\title{
ENTRE LA (RE)FRONTERIZACIÓN Y LA FRONTERA RED: PRÁCTICAS DE MOVILIDAD TRANSFRONTERIZA DE LOS INMIGRANTES DE LA EUROPA DEL ESTE EN ESPAÑA
}

\author{
Silvia Marcu \\ Instituto de Economía, Geografía y Demografía \\ Consejo Superior de Investigaciones Científicas \\ silvia.marcu@cchs.csic.es
}

\section{INTRODUCCIÓN Y OBJETIVOS}

El artículo examina las prácticas de movilidad transfronteriza de los inmigrantes de la Europa del Este (rumanos, búlgaros, moldavos y ucranianos) en las fronteras de la Unión Europea (UE), teniendo en cuenta los cambios en el régimen fronterizo de la UE que afectó la movilidad humana en los últimos 20 años. Basándose en la investigación empírica, con la realización de entrevistas en profundidad a inmigrantes de la Europa del Este en España, el artículo evidencia las vías en las que los modelos de movilidad transfronteriza producen nuevas geografías en las fronteras de la UE. Por un lado, se trata de la (re)fronterización que dificulta el movimiento humano, y por el otro, de la frontera red, que facilita la movilidad.

A pesar de que la frontera ha sido considerada como un elemento esencial de la movilidad, la investigación en el ámbito de las percepciones de los migrantes sobre el proceso de migración y movilidad transfronteriza no han sido analizados. Es lo que nos proponemos explorar en este artículo.

El primer objetivo es analizar la relación entre la movilidad transfronteriza y la ampliación de la UE hacia el Este, y destacar cómo la migración se transforma en movilidad siguiendo la política de la apertura de las fronteras en la UE ampliada.

El estudio examina tres periodos de la migración de los europeos del Este en Europa y España: el primer periodo (1992-2002), cuando la frontera permaneció cerrada para todos los grupos de migrantes estudiados; el segundo periodo (2002-2007), cuando las fronteras se abrieron para los rumanos y los búlgaros en el marco del Acuerdo Schengen (por un periodo de no más de tres meses) pero no para los moldavos y ucranianos; y el tercer periodo, que ha continuado desde 2007, tras la ampliación de la UE hacia Rumania y Bulgaria, que permitió 
la libre circulación a los ciudadanos, a pesar de que algunos países, restringieron la libertad de trabajo, hasta 1 de Enero de 2014. En el caso de los moldavos y ucranianos, siguen necesitando visas de entrada para viajar en el territorio de la UE.

El artículo intenta continuar la tesis de Rumford (2006), al examinar los mecanismos a través de los que la UE construye y reconstruye sus fronteras con los países vecinos, y cómo esos procesos son dinámicos, contingentes y a menudo contradictorios. Por lo tanto, el artículo capta cómo la UE opera con dos categorías de regímenes fronterizos, que pueden tener consecuencias en la (re)territorialización de las fronteras del continente. Mientras que en el marco de la UE, las fronteras ya no representan impedimentos para la movilidad, las fronteras externas se transformaron en obstáculos físicos para el movimiento de las personas (Scott y Houtum, 2009).

En segundo lugar, se analiza la importancia que tiene la superación de las fronteras para los ciudadanos móviles, mientras se destaca la movilidad entre la Europa del Este y la UE, los caminos en los que los migrantes y ciudadanos construyen sus experiencias de movilidad más allá de la frontera.

\section{APROXIMACIÓN TEÓRICA DE LA MOVILIDAD TRANSFRONTERIZA}

El proceso de (re)fronterización define un nuevo espacio cultural y conceptual de las fronteras de la UE (Paasi, 2009) e incluye las prácticas políticas y burocráticas legales establecidas en relación con los países que no forman parte de la UE y con el derecho de circulación y trabajo de sus ciudadanos. Otra vía para teorizar las fronteras en términos espaciales es la idea de frontera red, teorizada en los últimos años, como espacios de apertura y conexión (Dicken, Kelly, Olds y Yeung, 2001: 97; Rumford, 2007) y que favorecen la movilidad.

La tensión existente entre las fronteras red y la refronterización ha abierto la posibilidad de diferenciar las fronteras en la UE (Hassner, 2002). La tesis de la refronterización no puede acomodarse fácilmente a la permeabilidad de la frontera red, que tiene el valor de minimizar la importancia de la frontera cerrada.

El siglo XXI, sin embargo, se percibe como la era de la movilidad, fluidez, apertura y conectividad. Según Cooper y Rumford (2013: 108) en la era de la movilidad, las fronteras dejan de ser líneas divisorias; ellas delinean y conectan el interior con el exterior (Van Houtum, Kramsch y Zierhofer, 2005), formando espacios liminales. Como señala Paasi (2009: 45) las fronteras crean canales de paso para algunos y barreras para otros. En relación a ello, Balibar (2002) señala que el análisis de la movilidad transfronteriza revela la realidad de las políticas de la movilidad y sus dinámicas, especialmente las referidas a las regulaciones legislativas, la geopolítica de seguridad o las políticas de identidad y diferencia. En este contexto, las estrategias de la seguridad tienen que imaginarse a escala global. King, Skeldon y Vullnetari (2008:2) señalan que la distinción entre los movimientos internos e internacionales es cada vez más borrosa, no sólo debido a los acontecimientos geopolíticos y a la configuración de las fronteras, sino porque los viajes de los migrantes se multiplicaron y fragmentaron.

Como resultado de la nueva coreografía fronteriza, el transnacionalismo emergió como un espacio en el cual los migrantes se practican la movilidad, fundamentalmente, por razones de trabajo, intentando situarse continuamente entre el aquí y el allí (Glick Schiller, Basch y 
Blanc-Szanton, 1992; Portes, Guarnizo y Landolt, 1999), y jugando a la vez un papel activo en la configuración del espacio transnacional (Hannerz, 1996). Por lo tanto, la movilidad y la fluidez (Hannam, Sheller y Urry, 2006) permiten la conectividad a través de las fronteras de la EU (Rumford, 2007), su dependencia e interdependencia económica, la proximidad geográfica y la imposibilidad de que la UE se separe de sus vecinos. En este caso, las fronteras adquieren significados diferentes, transformándose en espacios de intercambio, interacción e integración.

Por otra parte, tal como señala Van Houtum (1999: 330) no solo es importante la realidad objetiva de las fronteras, sino también su realidad subjetiva, los sentimientos, las acciones, los pensamientos de los actores que se confrontan con ellas. Por lo tanto, debido a su porosidad las fronteras levantan muchas preguntas inquietantes: ¿Quién está al otro lado de la línea de división? ¿Cuál es el precio para superarla? ¿Me costará dinero, o tal vez me costará la vida? Todos estos miedos ponen en evidencia el significado de "límite" de la frontera. Es la aproximación realizada por las personas (Van Houtum, 1999) y se refiere a la interacción o a la falta de interacción entre la gente situada a los dos lados de la frontera. Por lo tanto, la movilidad de los europeos del Este a través de las fronteras puede percibirse como una construcción mental, un proceso de aprendizaje mental que comienza en el país de origen. La frontera se divide no sólo en el sentido espacial sino también en el sentido mental, y el efecto producido por "nosotros-ellos" nutre la distancia mental en la movilidad transfronteriza. Esta aproximación es, a menudo, utilizada en las investigaciones sobre la frontera en la UE. De Gisjel y Janssen (2000: 66) apuntan que "las distancias mentales juegan un papel importante en los mercados laborales de la UE". Por lo general, los estudios que se podrían categorizar como "aproximación de las personas" se mantienen lejos de la fijación de las fronteras como delimitaciones políticas en el espacio (Paasi, 1999), y se centran, sobre todo, en las reacciones emocionales de la gente que se confronta con la movilidad transfronteriza y cómo aprenden a tratar con la frontera a través de la movilidad. El concepto de "aprendizaje de la movilidad", sin embargo, ha sido poco estudiado en la literatura. Morokvasic (2004: 9) señala que la movilidad transfronteriza tiene una función de aprendizaje, mientras que (Vertovec (2007: 5) apunta que, una vez que las personas aprenden cómo cruzar la frontera, se preocupan menos si son legales o no. Si queremos comprender mejor la configuración y las características de la movilidad transfronteriza, debemos incluir en el análisis, las percepciones de las personas que la practican.

\section{METODOLOGÍA}

La investigación utiliza la metodología cualitativa que ayuda a comprender la complejidad de la conducta humana. Se realizaron 78 entrevistas en profundidad (2011 y 2012) en España (Comunidad de Madrid y Comunidad Valenciana) a hombres y mujeres en edad laboral, por este orden: 32 rumanos, 20 búlgaros, 14 moldavos y 12 ucranianos que llegaron al país en tres etapas: antes de la apertura de la frontera Schengen, tras la apertura, y después del ingreso en la UE de Rumania y Bulgaria.

Con el fin de añadir el elemento transfronterizo al trabajo empírico, y para obtener una mayor comprensión de las complejidades relacionadas con la experiencia de la estrategia de movilidad y retorno, además, se llevaron a cabo entrevistas a personas que retornaron de 
España a sus países, y a familiares de personas que emigraron a España (media de 15 entrevistas por país. Al mismo tiempo, se viajó a la frontera situada entre Rumania y Moldavia y se realizaron entrevistas en profundidad y trabajo de observación en Giurgiulesti y Albita, y en Cernauti, en la frontera situada entre Rumania y Ucrania. Además, se entrevistaron dos conductores de autobús que realizan trayectos regulares entre Ucrania - España y entre Rumania-España.

Las entrevistas fueron transcritas, (traducidas en su caso) codificadas y analizadas con el programa ATLAS Ti versión 6.2, de acuerdo con los datos que surgieron. Debido a la cantidad de información obtenida, se optó por utilizar el análisis temático que es un buen ejemplo de la técnica de reducción de datos para la investigación cualitativa. Se identificaron los temas de experiencia fronteriza (fronteras, viaje, entrada en España), ampliación de la UE e influencia sobre la movilidad. Para contestar a las preguntas de investigación, el estudio comienza con la suposición teórica de que los migrantes son activos en sus decisiones y experiencias de migración/movilidad transfronteriza.

\section{CRUZAR LAS FRONTERAS}

La actual experiencia de la movilidad de los europeos del este tiene sus raíces en el pasado dictatorial. Según recuerda Urry (2007:10) una parte importante de la movilidad humana sigue siendo altamente dependiente de pasaportes, visados permiso de residencia y cualificación de mano de obra. Como apunta Guild (2009) existen varias etapas en el proceso transfronterizo, y la primera es la aplicación para obtener un visado. Las reglas en torno a la obtención del visado se encuentran en la Instrucción Consular Común en virtud del Convenio de aplicación de Schengen y en la parte del Derecho comunitario (artículo 62 de la UE) que indica claramente que la capacidad económica del individuo es una consideración muy importante para la potencial concesión de visado.

Si indagamos en la legislación aplicada por igual a los nacionales de los cuatro países en la primera etapa, señalamos que para este periodo, hay pocas diferencias entre las trayectorias de los europeos del Este. Los migrantes utilizaron la estrategia de la migración irregular a través de la falsificación de pasaportes o mediante viajes turísticos.

El discurso de los entrevistados se construye alrededor del recuerdo, que ellos vinculan, en primer lugar, a los motivos de la salida. Los relatos llegan desde la memoria, y las voces reflejan el recuerdo de la angustia vivida, durante el procedimiento de obtener el visado y también en el momento de cruzar la frontera. Prácticamente todos viajaron por otros países europeos hasta llegar a España.

Los ucranianos tienen experiencia de viajar a varios países europeos como Austria o Alemania. Los moldavos destacan los engaños sufridos y las pérdidas de dinero en los intentos de obtener el visado. Sin embargo, todos los entrevistados destacan la flexibilidad de las fronteras en los controles de entrada por carretera o tren, en España.

Con todo, se puede señalar que desde 1990, el antiguo telón de acero estuvo reemplazado por el "muro de papel" alzado por la necesidad de visado, invitaciones o declaraciones consulares.

Sin embargo, el deseo de aprender de las experiencias de la primera etapa de la movilidad, ofrece una gran ayuda en la búsqueda de mecanismos para eludir estos muros de papel. 


\section{ENTRE LA FRONTERA-RED Y LA (RE)FRONTERIZACIÓN}

En la segunda etapa, que situamos entre 2002 y 2007, nos encontramos con la apertura fronteriza en la UE, que abre el paso hacia la movilidad de las personas. Nos referiremos por separado a las dos categorías de casos:

1) En primer lugar, situaremos a los rumanos y los búlgaros que, con el apoyo de la legislación europea de apertura del espacio Schengen, crean redes de movilidad circulatoria laboral. Desafían las fronteras, aún sabiendo que no pueden viajar si no demuestran que tienen una cantidad de dinero que les ayude a sufragar los gastos diarios de su estancia en España. En comparación con la primera etapa cuando la gente no sabía exactamente hacia donde finalizaba su viaje, en esta etapa, se viajó, sabiendo que algún familiar, amigo o conocido, les esperaba. Para los rumanos y búlgaros, las circunstancias cambiaron; los migrantes se transforman, gradualmente, en ciudadanos que circulan. Una vez llegados a España, ellos tienden a insertarse más fácilmente en la sociedad de acogida. Debido a los vínculos que mantienen con sus países, y a la facilidad de moverse, consiguen crear una cierta fluidez de movimiento y hacer frente a la dificultad de cruzar la frontera. He aquí como el transnacionalismo y la creación del espacio transnacional apoyan al ciudadano que practica la movilidad, a aprender a convivir con la frontera. Siguió habiendo turbulencia e inseguridad, porque los rumanos y búlgaros sólo podían permanecer por un periodo de tres meses en España, y en la frontera se les exigía continuamente dinero, ya sea por demostrar que podían sufragar los gastos de manutención en España, ya sea por la corrupción de los conductores de autobús, o de los funcionarios en los puestos de control fronterizos. Según las entrevistas, hubo casos de corrupción y extorsión en varios puntos de cruce fronterizo de la Europa del Este. La legislación española, a su vez, apoyó el proceso de movilidad mediante la regularización extraordinaria que se realizó en 2005, y mediante la cual se regularizaron más de 600.000 personas en España, una gran parte de ellos, ciudadanos de los países del Este de Europa. Concretamente, un 30\% de los entrevistados para este trabajo obtuvo sus papeles en España, a raíz de esta regularización extraordinaria.

2) En segundo lugar, observamos cómo los entrevistados moldavos y ucranianos aprenden a vivir con la frontera. Sus experiencias cambiaron. La semiapertura fronteriza, para los que pueden solicitar y obtener ciudadanía europea, les confiere el estatuto de ser móviles, y, de esta manera, aprenden que para emigrar, hay que arriesgar, aprenden la lección del viaje transfronterizo.

Los entrevistados que no ostentan ciudadanía europea, destacan las dificultades, pero en esta etapa del viaje, aprenden nuevas estrategias, como viajar solos, mezclarse con personas de la sociedad de acogida para pasar, si fuera preciso, inadvertido.

\section{VI. ¿HACIA LA MOVILIDAD A TRAVÉS DE LA CONECTIVIDAD DE LA FRONTERA?}

Para los entrevistados rumanos y búlgaros de la tercera etapa, la frontera, en la mayor parte, pasa inadvertida.

A partir de 2007, con el ingreso de sus países en la UE los rumanos y los búlgaros disminuyeron sus viajes en autobús y prefieren viajar el avión, aprovechando las líneas de bajo coste como Air Blue. Los trayectos se multiplicaron desde las ciudades más importantes 
de Rumania y Bulgaria, hasta las ciudades españolas, donde se afincó el mayor número de rumanos y búlgaros. Por otra parte, los inmigrantes procedentes de Rumanía y Bulgaria experimentaron una movilidad ascendente; muchos se asentaron con sus familias en España y tienen hijos que pertenecen a la segunda generación. Estos ciudadanos se integraron en las estructuras locales, y esto se refleja en las redes sociales creadas.

Los moldavos y ucranianos que tienen ciudadanía europea, por su lado, destacan la flexibilidad de la frontera española en comparación con otras fronteras europeas. Los ucranianos siguen llegando a España en su mayor parte, como turistas, con pasaportes falsos, que adquirieron en el mercado de pasaportes falsos de Kiev o de Odessa.

Es importante señalar que, desde el comienzo de la recesión, España experimentó un enorme aumento en el desempleo, sobre todo en el caso de los inmigrantes y de los trabajadores temporales, que sufrieron las consecuencias en la alta tasa de desempleo. Con todo, la movilidad facilitada para los ciudadanos comunitarios, abre la perspectiva de aproximación a la UE, y de la necesidad que tienen los ciudadanos que se mueven de sentirse europeos.

\section{CONCLUSIONES}

El artículo destacó la importancia de la frontera, a raíz de las prácticas de movilidad de los migrantes de la Europa del este, que a lo largo de los últimos 20 años cruzaron las fronteras y llegaron a España. En un ámbito teórico, y debido a la política de apertura de la frontera europea, el artículo defendió la contribución del estudio fronterizo en la comprensión de la movilidad humana. Contestando a las preguntas de investigación que planteamos al comienzo de este artículo, podemos sacar las siguientes conclusiones:

1) La frontera representa más bien un recurso que un obstáculo para la movilidad humana actual, en su doble ambivalencia de puente y barrera.

2) En segundo lugar, pudimos comprobar cómo la flexibilidad de las fronteras europeas facilitó la movilidad humana del este de Europa.

3) Finalmente, el artículo indagó en las formas de comprensión de los europeos del Este en cuanto la movilidad transfronteriza. De esta manera, se pudo observar el cambio en la percepción fronteriza de los europeos del Este que practicaron la movilidad: Si los migrantes de la primera etapa tenían que enfrentarse a las fronteras por necesidad, los de la segunda ola de movilidad, con el apoyo de las redes transnacionales creadas en el destino, aprenden a convivir con ella, mientras que los de la tercera etapa aprenden la movilidad, y la practican, en su condición de ciudadanos europeos. Pudimos comprobar, asimismo, que si para los rumanos y búlgaros, a partir de 2002, y sobre todo desde 2007, la experiencia de cruzar la frontera se convirtió en un viaje, para los moldavos y ucranianos, sigue siendo un aprendizaje de superación. 